\title{
REVIEW
}

\section{Methods for studying tooth root cementum by light microscopy}

\author{
Brian L Foster
}

The tooth root cementum is a thin, mineralized tissue covering the root dentin that is present primarily as acellular cementum on the cervical root and cellular cementum covering the apical root. While cementum shares many properties in common with bone and dentin, it is a unique mineralized tissue and acellular cementum is critical for attachment of the tooth to the surrounding periodontal ligament (PDL). Resources for methodologies for hard tissues often overlook cementum and approaches that may be of value for studying this tissue. To address this issue, this report offers detailed methodology, as well as comparisons of several histological and immunohistochemical stains available for imaging the cementum-PDL complex by light microscopy. Notably, the infrequently used Alcian blue stain with nuclear fast red counterstain provided utility in imaging cementum in mouse, porcine and human teeth. While no truly unique extracellular matrix markers have been identified to differentiate cementum from the other hard tissues, immunohistochemistry for detection of bone sialoprotein (BSP), osteopontin (OPN), and dentin matrix protein 1 (DMP1) is a reliable approach for studying both acellular and cellular cementum and providing insight into developmental biology of these tissues. Histological and immunohistochemical approaches provide insight on developmental biology of cementum. International Journal of Oral Science (2012) 4, 119-128; doi:10.1038/ijos.2012.57; published online 21 September 2012

Keywords: bone; bone sialoprotein; cementum; dentin; dentin matrix protein 1; osteopontin; periodontal ligament; tooth development

\section{INTRODUCTION}

The tooth and its supporting tissues are unique by virtue of being home to four distinct mineralized tissues, the enamel, dentin, tooth root cementum and alveolar bone, and their associated hard-hard and hard-soft tissue interfaces (Figure 1). The tooth root cementum is a thin, mineralized tissue covering the root dentin surface. Cementum is primarily present in two varieties, the acellular extrinsic fiber cementum (AEFC, acellular or primary cementum) and cellular intrinsic fiber cementum (CIFC, cellular or secondary cementum), though mixed stratified cementum exhibits layers of both types in some species. ${ }^{1-6}$ Acellular cementum covering the cervical portion of the root is critical for tooth attachment to the adjacent periodontal ligament (PDL), while cellular cementum covering the apical root is hypothesized to play a role in post-eruptive tooth movement and adaptation to occlusion. Mixed stratified cementum includes layers of both acellular and cellular types, and is present on apical roots or in the furcation region of some species. Cementum is likely the least understood of the mineralized tissues of the skeleton and dentition, with little known of the signaling pathways involved in cementogenesis, and debate still ongoing on the origin and lineage of cementoblast cells. Developmental differences in cementum versus bone, and even between AEFC and CIFC remain unclear. ${ }^{3,6}$ Some authors have suggested cementoblasts are positional osteoblasts and cementum a type of bone, though cementum differs from bone by virtue of its being avascular, non-innervated, and not subject to remodeling or physiological turnover.
Rodent models have commonly been used to study cementum, and transgenic mice in particular have been of great value in identifying critical regulators of cementum formation. However, the study of mouse cementum is technically challenging in that dimensions of this tissue are very small in rodents, and stages of tooth development proceed rapidly compared to humans and other large animal models. While there are several excellent resources for staining methodology for hard tissues, ${ }^{7-8}$ these often focus on bone and cartilage and do not directly address cementum and the approaches that may be of value for specifically studying that tissue. This report focuses on techniques useful for the study of cementum that can be applied using light microscopy. Additional imaging methods that may be of use for examining this tissue include scanning and transmission electron microscopy, as well as other approaches such as micro-computerized tomography (microCT), that may be limited by resolution and animal model used (i.e., size of cementum and ability to detect it). This report will focus on methods for the distinction of acellular and cellular cementum and differentiating them from nearby mineralized tissues, dentin and bone, and from the attached PDL. Several histological stains will be presented, as well as immunohistochemical techniques, and detailed methodology is provided for each approach.

\section{MATERIALS AND METHODS}

Animals and human samples

Mice with background of CD1 or mixed background of 129S1/SvImJ or $\mathrm{C} 57 \mathrm{BL} / 6$ were used for histological and immunohistochemical 


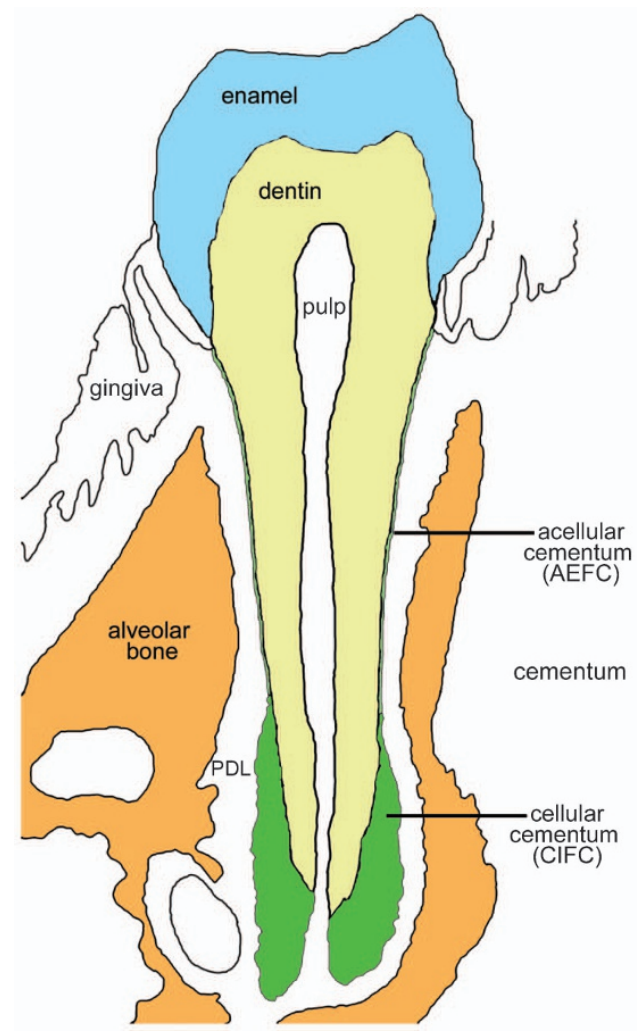

Figure 1 Schematic of the mouse molar tooth. The illustrated image is modeled after a coronal buccolingual section of a mouse first mandibular molar, and the mineralized tissues of the dentoalveolar are emphasized by color enhancement: enamel (blue), dentin (yellow), cementum (green for both acellular and cellular varieties) and alveolar bone (orange). The pulp chamber, PDL and gingiva are also labeled as relevant soft tissues. AEFC, acellular extrinsic fiber cementum; CIFC, cellular intrinsic fiber cementum; PDL, periodontal ligament.

staining studies. Mice null for tissue nonspecific alkaline phosphatase (gene $A l p l$ ) were employed as a model featuring deficient cementum formation. ${ }^{9} \mathrm{Alpl}^{-1-}$ mice were prepared and genotyped as previously described. ${ }^{9-10}$ Normal mice were allowed access to water and standard rodent chow ad libitum, while Alpl mice were fed a rodent diet supplemented with pyridoxine (vitamin $\mathrm{B}_{6}$ ) to suppress seizures in homozygous null pups. Hanford miniature pigs were employed to harvest first mandibular molar tissues at 13-16 weeks of age, as described previously. ${ }^{11}$ Mouse tissues were harvested at the University of Washington (Seattle, WA, USA) or Campinas State University (Piracicaba, SP, Brazil), and pig tissues were harvested as University of Washington. All animal procedures were approved by the respective institutional committees, the Institutional Animal Care and Use Committee (University of Washington) or Campinas Institutional Animal Care and Use Committee (Campinas State University). Human samples included de-identified teeth extracted at the University of Pittsburgh School of Dental Medicine (Pittsburgh, PA, USA). Teeth were removed for orthodontic reasons, and use of deidentified discarded dental tissues for research activities was approved at University of Pittsburgh and the National Institutes of Health (Bethesda, MD, USA).

Tissue fixation, decalcification, processing and paraffin embedding For decalcified histology and immunohistochemistry used here, mouse heads/mandibles and pig tissues were fixed in Bouin's solution
(Electron Microscopy Sciences, Hatfield, PA, USA) for 18-24 h, and then stored in $70 \%$ ethyl alcohol at $4{ }^{\circ} \mathrm{C}$, unless otherwise noted. Human teeth were fixed in 10\% neutral buffered formalin. Mouse samples of age 8 days postnatal ( $\mathrm{dpn}$ ) or older were decalcified in acid flagellates solution (AFS): $100 \mathrm{~mL}$ glacial acetic acid, $40 \mathrm{~mL} 10 \%$ neutral buffered formalin, $8.5 \mathrm{~g} \mathrm{NaCl}$, brought to $1.0 \mathrm{~L}$ with deionized water. Samples in AFS were loaded into labeled histology cassettes and agitated in beakers by magnetic stir bar at $4{ }^{\circ} \mathrm{C}$ for $2-3$ weeks until incisor tooth was flexible by manual testing. Pig molar tissues were decalcified sequentially in $8 \%$ ethylenediaminetetraacetic acid (EDTA) and then $25 \%$ formic acid, as previously described. ${ }^{11}$ Human molar teeth were hemisected and decalcified in $20 \%$ EDTA at $4{ }^{\circ} \mathrm{C}$ for approximately 5 weeks, until flexible by manual testing. Mouse tissues used for dentin matrix protein 1 (DMP1) immunohistochemistry (see details below) were fixed in neutral buffered formalin and decalcified in $20 \%$ EDTA at $4{ }^{\circ} \mathrm{C}$. Decalcified tissues were processed by automatic tissue processor (Tissue-Tek 3 000; Sakura, Torrance, CA, USA or ASP 300; Leica Microsystems, Buffalo Grove, IL, USA), and embedded in paraffin in base molds for manual serial sectioning at a thickness of $5 \mu \mathrm{m}$ for mouse and human, and $7 \mu \mathrm{m}$ for pig. Coronal (buccolingual) sections of mouse molars, and mesiodistal sections of pig and human molars are shown in the section on 'Results'. For techniques evaluated in this paper, specific ages for mice were chosen as representative illustrations; however, methods described were used in numerous samples of different ages and genetic backgrounds over the past several years, and references to this effect are provided whenever possible.

While there are many options for tissue fixatives, our laboratory has routinely used Bouin's solution, which has provided high-quality morphology, generally intense immunohistochemical reactions, and also allowed in situ hybridization detection of mRNA all in the same paraffin-embedded tissue blocks, allowing efficient use of collected tissues. It should be noted, however, that Bouin's solution is acidic and will begin demineralization of teeth and bones, making it a poor choice for tissues to be first imaged by radiography or micro-computerized tomography, for instance. There are also several choices and variations for decalcifying hard tissue specimens, including EDTA, AFS and formic acid, in order of slowest to fastest rate of decalcification. EDTA and AFS both offer the ability to perform immunohistochemistry and in situ hybridization, in addition to histology.

\section{Histological staining}

Three histological stains were compared for cementum imaging in decalcified sections, and one additional stain technique was used for visualizing the cementum/PDL interface and PDL organization. For all histological staining of decalcified sections described below, tissues were deparaffinized in xylene for $2 \times 8 \mathrm{~min}$, rehydrated in a descending ethanol series ( $100 \% 2 \times 5 \mathrm{~min}, 90 \%$ for $5 \mathrm{~min}, 80 \%$ for $5 \mathrm{~min}, 70 \%$ for $5 \mathrm{~min}$ and $50 \%$ for $5 \mathrm{~min}$ ) and rinsed in deionized water $2 \times 5 \mathrm{~min}$. For hematoxylin and eosin (H\&E) staining, deparaffinized and rehydrated sections were dipped in hematoxylin for $20 \mathrm{~s}$, placed under gently running water for $10 \mathrm{~min}$, dipped in eosin Y for $4 \mathrm{~min}$, placed under gently running water for more than $10 \mathrm{~min}$, then dehydrated in an ascending ethanol series ( $50 \%$ for $5 \mathrm{~min}, 70 \%$ for $5 \mathrm{~min}, 80 \%$ for $5 \mathrm{~min}$, $90 \%$ for $5 \mathrm{~min}$ and $100 \% 2 \times 5 \mathrm{~min}$ ), then cleared in clean xylene $2 \times 10 \mathrm{~min}$ and allowed to air dry for $60 \mathrm{~min}$ before mounting cover slips with toluene-based mounting solution, Permount (Fisher Scientific, Pittsburgh, PA, USA).

For toluidine blue (TB) staining, deparaffinized and rehydrated sections were placed in $0.1 \% \mathrm{~TB} \mathrm{O}$ solution for $3 \mathrm{~min}$, rinsed in deionized water $3 \times 5 \mathrm{~min}$ with gentle agitation, then dehydrated 
quickly through $90 \%$ ethanol (10 dips) and 100\% ( $2 \times 10$ dips). Sections were cleared in clean xylene $(2 \times 10 \mathrm{~min})$ and cover slips mounted with Permount. TB solution was purchased from Electron Microscopy Sciences.

For Alcian blue with nuclear fast red (AB-NFR) staining, deparaffinized tissues were placed in $1 \%$ Alcian blue in 3\% acetic acid solution ( $\mathrm{pH}$ 2.5) for $30 \mathrm{~min}$, placed under gently running water for $5 \mathrm{~min}$, counterstained in a solution of $0.1 \%$ nuclear fast red and $5 \%$ potassium alum for $5 \mathrm{~min}$, then dehydrated through an ascending ethanol series, cleared in xylene, and mounted with Permount, as described above. Alcian blue and nuclear fast red solutions were purchased from Electron Microscopy Sciences.

For Picrosirius red (PR) staining, deparaffinized sections were placed in $0.2 \%$ aqueous solution of phosphomolybdic acid hydrate for $2 \mathrm{~min}$, rinsed in deionized water $5 \mathrm{~min}$ with gentle agitation, placed in an aqueous solution of $0.4 \%$ Direct Red 80 and 1.3\% 2,4,6trinitrophenol for $60 \mathrm{~min}$, then placed directly in $0.4 \% \mathrm{HCl}$ for $2 \mathrm{~min}$, followed by a dehydration series in ascending ethanol, clearing in xylene, and cover slip mounting using Permount (as described above). Solutions for PR staining were purchased from Polysciences, Inc. (Warrington, PA, USA).

\section{Immunohistochemistry}

Immunohistochemistry (IHC) was performed on paraffin sections using an avidin-biotinylated peroxidase enzyme complex-based kit (Vectastain Elite; Vector Labs, Burlingame, CA, USA) with a 3amino-9-ethylcarbazole substrate (Vector Labs) to produce a red reaction product indicating antigen localization. Tissues were deparaffinized in xylene for $2 \times 8 \mathrm{~min}$, incubated in acetone at $-20{ }^{\circ} \mathrm{C}$ for $10 \mathrm{~min}$, rehydrated in a descending ethanol series $(100 \% 2 \times 5 \mathrm{~min}$, $90 \%$ for $5 \mathrm{~min}, 80 \%$ for $5 \mathrm{~min}, 70 \%$ for $5 \mathrm{~min}$ and $50 \%$ for $\mathrm{min}$ ), rinsed in deionized water $2 \times 5 \mathrm{~min}$, incubated in $3 \%$ hydrogen peroxide in methanol for $60 \mathrm{~min}$ while protected from light (to quench endogenous peroxidase), rinsed in phosphate-buffered saline (PBS) with $0.1 \%$ Tween for $5 \mathrm{~min}$, blocked with goat serum in $\mathrm{PBS}+0.1 \%$ Tween for $60 \mathrm{~min}$, then incubated with primary antibodies diluted in goat serum $+\mathrm{PBS}+0.1 \%$ Tween at $4{ }^{\circ} \mathrm{C}$ overnight. On the second day of IHC, slides were washed in PBS $+0.1 \%$ Tween $3 \times 15$ min with agitation, incubated with biotinylated secondary antibody for $2 \mathrm{~h}$, washed in PBS $+0.1 \%$ Tween $3 \times 15$ min with agitation, incubated with avidin-biotinylated peroxidase enzyme complex for $1 \mathrm{~h}$, washed in PBS $+0.1 \%$ Tween for $5 \mathrm{~min}$, then incubated with 3-amino-9-ethylcarbazole until desired color intensity was reached (typically 6-8 min for antibodies used here). Counterstaining was performed by dipping slides in hematoxylin for $20 \mathrm{~s}$ and then placing under gently running water for $10 \mathrm{~min}$, followed by cover slipping with aqueous mounting solution (EMS-Mount; Electron Microscopy Sciences, Hatfield, PA, USA). Primary antibodies included polyclonal rabbit anti-mouse bone sialoprotein (BSP; Dr Renny Franceschi, University of Michigan, Ann Arbor, MI, USA ${ }^{9,12-13}$ ), LF-175 rabbit anti-mouse osteopontin (OPN; Dr Larry Fisher, NIDCR, Bethesda, MD, USA ${ }^{9,12}$ ) and polyclonal rabbit anti-rat (N-terminal) DMP1 (Takara Bio, Mountain View, CA, USA ${ }^{9,12}$ ).

\section{Microscope, camera and software}

Slides were examined and images captured on an Axio Imager D2 (Carl Zeiss Microscopy, LLC, Thornwood, NY, USA) fitted with an AxioCam MRc5 digital color camera, and connected to a computer running AxioVision software version 4.8.2.0. PR staining was observed under polarized light on the same microscope, using a rotatable stage to confirm maximum signal intensity of collagen birefringence. Adobe Photoshop Elements 10 software was use for optimizing image levels.

\section{RESULTS AND DISCUSSION}

\section{Histological stains for cementum}

Serial sections of the mouse first mandibular molar at age 44 dpn were used to compare several histological stains. Ability for staining techniques to allow visualization of acellular and cellular cementum in the mouse was ascertained.

$H \& E$ staining. $\mathrm{H} \& \mathrm{E}$ staining is perhaps the most common differential stain for histological sections, and is widely used in other applications such as pathology biopsies to provide contrast to colorless cells and tissues for the purpose of studying morphology. In this pairing, hematoxylin carries properties resembling a basic dye and stains cell nuclei intensely blue, and other anionic cell and tissue components varying shades of blue. Eosin $\mathrm{Y}$ is classical acidic dye that counterstains cytoplasm and extracellular fibers pink, and red blood cells intensely red. $\mathrm{H} \& \mathrm{E}$ staining of decalcified and paraffin-embedded sections of the tooth and associated tissues revealed that the dentin, bone and cellular cementum are weakly basophilic (staining light pink to light bluepurple), while soft tissues of the pulp and PDL were evident by the intense blue stain of the numerous cell nuclei over a pink background (Figure $2 \mathrm{a}-2 \mathrm{~d}$ ). The AEFC of the cervical root presented as a basophilic layer formed on the root dentin surface (Figure 2d). H\&E staining allowed adequate contrast between acellular cementum and the underlying mantle dentin due to the basophilia of AEFC compared to the whitish or light pink staining of the mantle dentin directly underlying the cementum layer. The strong basophilic nature of the AEFC likely arises from the concentration of acidic extracellular matrix (ECM) proteins in this layer (e.g., bone sialoprotein, osteopontin, etc., described in more detail below). CIFC, on the other hand, stained similarly to bone with application of H\&E (Figure $2 b$ ), reflecting less concentration of acidic ECM proteins accompanying its rapid formation. ${ }^{3,6}$ While $\mathrm{H} \& \mathrm{E}$ provides good contrast between AEFC and dentin, it is often difficult to determine the precise border between CIFC and the underlying apical dentin.

TB staining. TB is a basic dye, commonly used without counterstain, for rapid and easy evaluation of morphology. Additionally, TB may interact with some tissue components that shift the color to red or purple, a property called metachromasia. TB staining of the dentoalveolar complex colored all of the tissues and cells varying intensities of blue. Most intense staining was seen in the circumpulpal dentin, marrow and gingiva (Figure $2 \mathrm{e}-2 \mathrm{~h}$ ). As with $\mathrm{H} \& \mathrm{E}$, cell nuclei stained blue, highlighting PDL and pulp cells, and embedded osteocytes of the bone. AEFC stained a dark blue, distinct from the whitish color of the mantle dentin, but similar in hue to the nearby cementoblast and PDL cell nuclei (Figure $1 \mathrm{~h}$ ). Like bone, cellular cementum stained a pale blue (Figure 2f), distinguished from the underlying dentin by the color difference and the visualization of the cementum-dentin junction (CDJ). Indeed, TB staining has been used to visualize mouse CIFC in transgenic mouse models, ${ }^{14}$ as well as to explore the CDJ in human teeth. ${ }^{15}$ TB staining does not offer many advantages for imaging AEFC, though the contrast between CIFC and dentin is markedly improved compared to H\&E.

AB-NFR staining. Alcian blue stain with nuclear fast red counterstaining (AB-NFR) provided excellent contrast for the acellular cementum layer in mice, which stained a brilliant and intense blue color, while the 

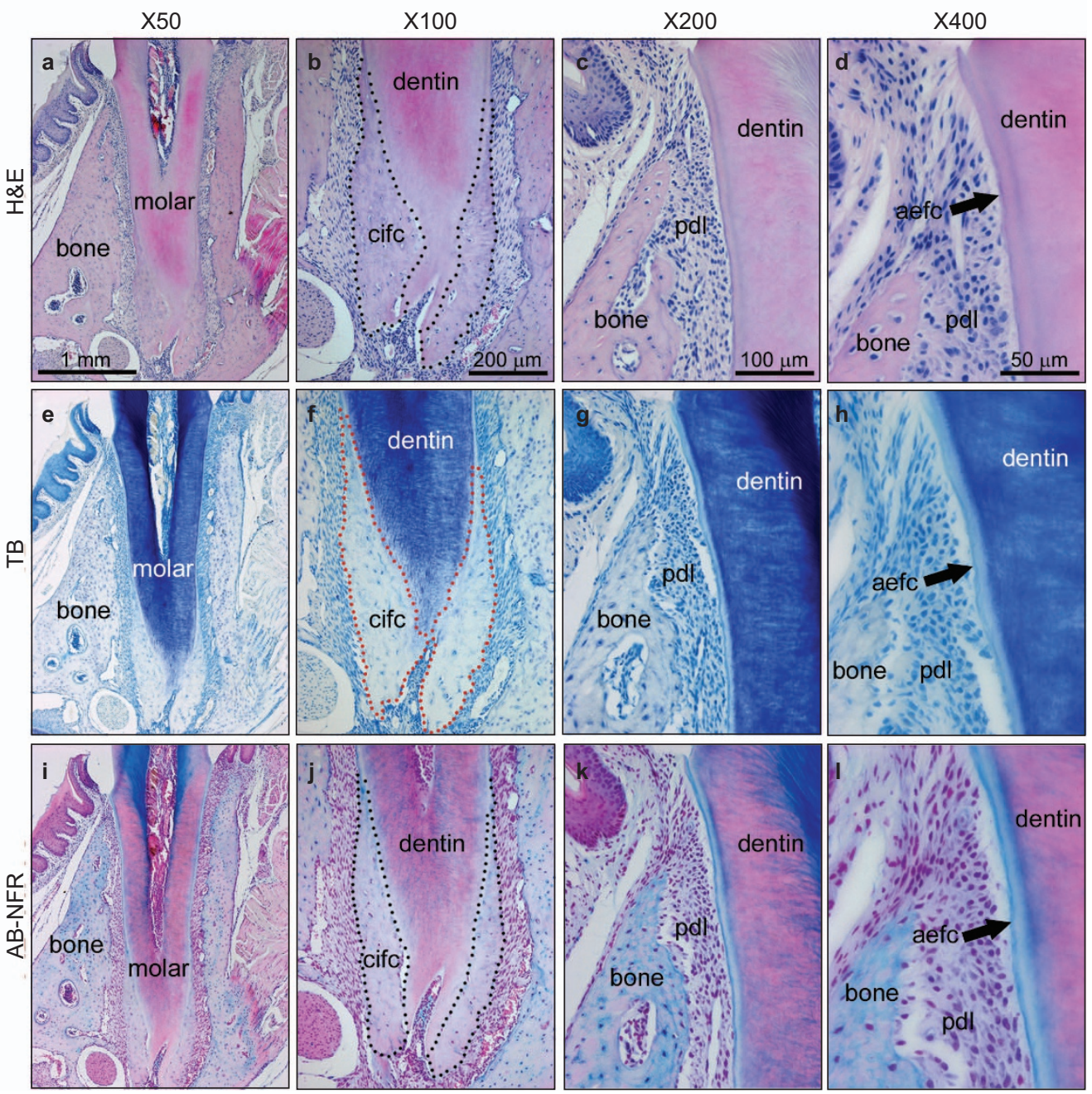

Figure 2 Histological stains for cementum. Serial sections of the 44 dpn mouse mandibular first molar were stained with (a-d) H\&E, (e-h) TB and (i-I) AB-NFR. Visualization of AEFC and CIFC is emphasized in the accompanying text. A dotted line is used to highlight the circumference of the CIFC in panels (b), (f) and (j). Original magnifications $(\times 50, \times 100, \times 200$ and $\times 400)$ are labeled over the panels. AB-NFR, Alcian blue and nuclear fast red; AEFC, acellular extrinsic fiber cementum; CIFC, cellular intrinsic fiber cementum; dpn, days postnatal; H\&E, hematoxylin and eosin; TB, toluidine blue.

adjacent PDL exhibited bright pink cell nuclei and pale pink cell cytoplasm, and the mantle dentin underlying the cementum is a pale blue that is readily distinguished from AEFC (Figure 2i-2l). Bone and cellular cementum also stained a pale blue with some pale pink staining of inserted collagen fibers. Dentin stained pink with intense blue coloration in circumpulpal regions of the crown and root (away from the cementum layer), also featuring blue stain that followed the paths of dentinal tubules and provided some fine details of dentinal tubule branching not visible with the other stains evaluated. This stain is superior to H\&E and TB by virtue of creating improved blue-to-pink contrast between the AEFC and the immediately adjacent cells and tissues, and also allows easy distinction of cellular cementum from dentin. Despite the notable advantages of AB-NFR, variations on this approach have rarely been reported in cementum investigations. ${ }^{16}$ Alcian blue stains acidic mucopolysaccharides, which may include sulfated and glycosylated glycoproteins like small integrin binding ligands N-linked glycoprotein family members BSP and OPN ${ }^{17-18}$ that are concentrated in AEFC, and would also include proteoglycans. The strong blue reaction in the AEFC is more likely to result from the concentrated deposition of acidic ECM proteins on the root surface, because it has been reported that AEFC proper contains little or no proteoglycan content, although the PDL, CIFC, bone and dentin are proteoglycan-rich. ${ }^{1,3,6}$

PR staining viewed under polarized light. One of the key functional characteristics of the acellular cementum is the insertion of collagen fibers from the PDL, the so-called Sharpey's fibers. ${ }^{3,6,19} \mathrm{PR}$ is a strong acidic dye that is thought to stain collagen by reacting with basic amino-acid groups in the collagen molecule. Junqueira and colleagues demonstrated that PR staining, when combined with polarized light microscopy, enhanced the natural birefringency of fibrillar collagen, and could be considered a relatively specific dye for collagen as a result. ${ }^{20}$ The highly organized collagen fibers of the PDL give an intense signal under polarized light after PR staining, and thus this staining approach has been used to analyze organization and angle of PDL fiber groups and orientation of fibers near the tooth root surface. ${ }^{9,21-22}$ In Figure 3, PR-stained tissues are shown under polarized light (to show collagen fiber organization) in Figure 3a-3d, and non-polarized light (to show overall tissue morphology, including non-birefringent tissues) in Figure 3e-3h. In Figure 3a-3d, the PDL 

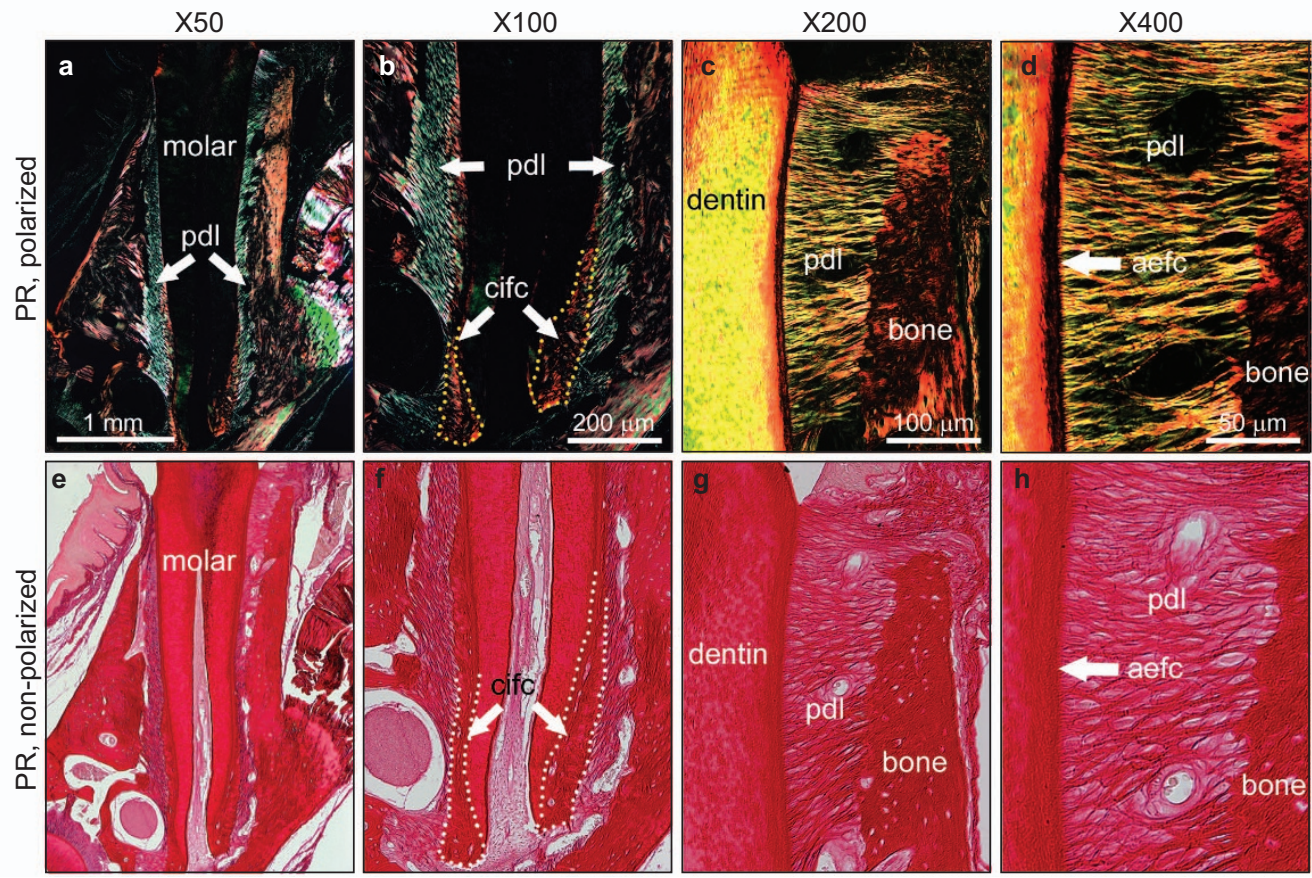

Figure 3 PR staining of the cementum-periodontal ligament complex. Mouse first molar sections at $44 \mathrm{dpn}$ stained by the PR method were viewed under (a-d) polarized light to visualize AEFC and CIFC and collagen organization and insertion from the PDL. The same sections under (e-h) non-polarized light are displayed to indicate overall tissue morphology. A dotted line is used to highlight the circumference of the CIFC in panels (b) and (f). Original magnifications ( $\times 50, \times 100, \times 200$ and $\times 400$ ) are labeled over the panels. AEFC, acellular extrinsic fiber cementum; CIFC, cellular intrinsic fiber cementum; dpn, days postnatal; PDL, periodontal ligament; $\mathrm{PR}$, Picrosirius red.

can be seen to display intense yellow staining derived from its strong birefringence, i.e., organized nature and parallel structure of the collagen fibers. PR staining showed that the PDL collagen fibers clearly extended from the alveolar bone to the tooth root surface in a highly organized fashion, and made visible the Sharpey's fibers inserting through the acellular cementum give that tissue its namesake 'extrinsic fibers' (Figure 3d). The cellular cementum can be seen in Figure 3b, where red-colored collagen fibers within the CIFC appeared to be largely discontinuous with PDL collagen fibers, reflecting that tissue's intrinsic fibers.

It has been observed that different types of collagen display different interference colors under PR staining and polarized light. ${ }^{23}$ Collagen type I forms thick fibers of closely packed fibrils and displays a brilliant yellow or red color under polarized light, while collagen type III forms thin fibers out of loosely packed thin fibrils and displays a green color. Yellow fibers predominate in PR staining of the PDL, but less intense green fibers are also apparent, consistent with the major collagen types being I and III, with lesser amounts of types IV, VI and XII reported. ${ }^{24}$ The interference colors and intensity have been shown to be due to different patterns of physical aggregation of fibrils in different types of collagen, ${ }^{23}$ and with consistent sectioning, this effect can be predictable, though care should be taken not to over-interpret coloration as a specific interaction of PR stain with specific collagen types.

Another note of caution concerns the specificity of PR stain for collagen; PR dye staining patterns alone do not specifically or selectively interact with collagen, as can be seen under non-polarized light in Figure $3 \mathrm{e}$ where all structures stained pink to red when PR was applied. It is essential to observe the PR-stained section under polarized light and rotate the slide (a rotatable microscope stage is the easiest way to do so) to confirm that birefringence is enhanced (i.e., signal is intensified) by polarized light. ${ }^{20}$ Rotating the tissue specimen under polarized light will reveal that the birefringent signal will vary from a maximal intensity to near extinguishment depending on the orientation of the collagen fibers compared to the angle of the polarizer. A good approach for studying the birefringence of a particular collagen-rich area is to rotate the stage until maximum signal is achieved in that local region, as was done for higher magnification (Figure $3 \mathrm{c}$ and $3 \mathrm{~d}$ ).

Application of histological stains for detection of cementum defects. Analysis of potential cementum defects in mouse models can be technically challenging, especially at early developmental ages when the AEFC has just begun forming on the root surface, and is quite thin (about $1-2 \mu \mathrm{m}$ ). The alkaline phosphatase null $\left(\mathrm{Alpl}^{-/-}\right)$mouse was used as a model for acellular cementum deficiency $y^{9,25-26}$ in order to test the capability of ABNFR and PR for detection of cementum-PDL disruption. The Alpl $\mathrm{P}^{-1-}$ mouse is a model for the infantile form of hypophosphatasia, and inherited loss-of-function of tissue nonspecific alkaline phosphatase. ${ }^{10,27}$ Hypophosphatasia patients suffer from defective skeletal mineralization, and often prematurely lose deciduous and permanent dentition due to defective cementum development and the resultant poor periodontal attachment. $^{28-29}$ Whereas AB-NFR revealed the thin AEFC layer in wild-type (WT) control mouse molar teeth (Figure $4 \mathrm{a}$ and $4 \mathrm{~b}$ ), $A l p I^{-l-}$ mice featured a clear loss of the blue-stained acellular cementum layer at $14 \mathrm{dpn}$. Further, the orderly PDL collagen fiber arrangement of the WT molar that was evident by PR staining under polarized light was severely disrupted in the $\mathrm{Alpl}^{-1-}$ mouse molar root, leading to PDL detachment consistent with tooth loss in hypophosphatasia patients. Thus, the combination of approaches targeting cementum imaging and assessment of PDL organization revealed the crux of the periodontal developmental defect in this model. 


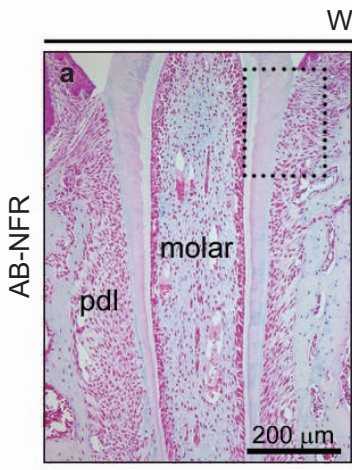

WT
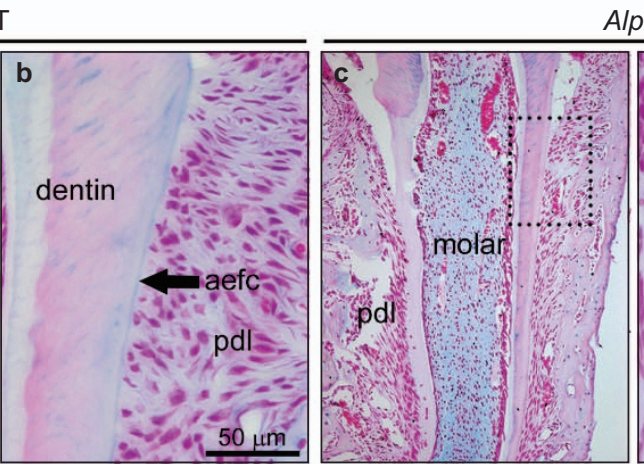

$A / p I^{-/-}$
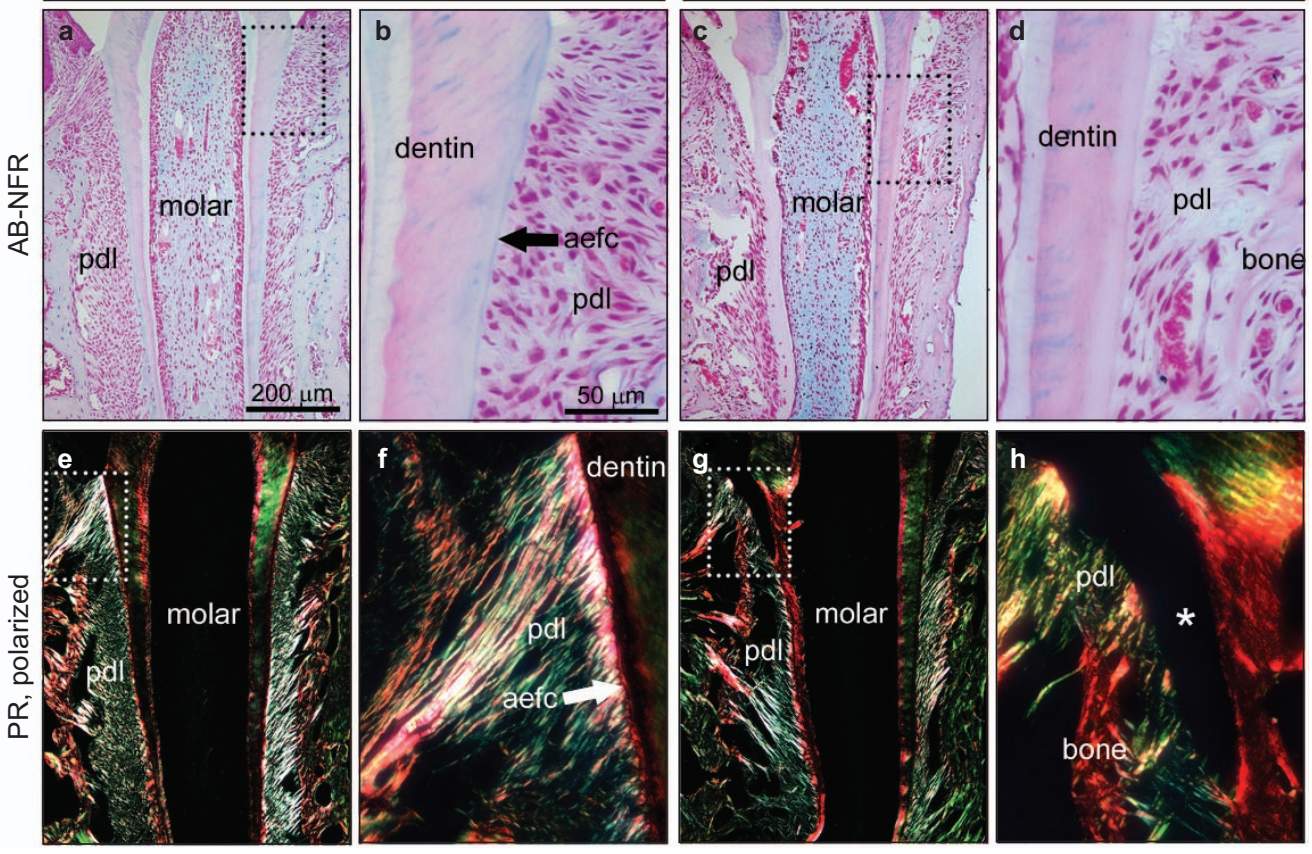

Figure 4 Staining to detect cementum-periodontal ligament defects in the alkaline phosphatase deficient mouse. WT and alkaline phosphatase null $\left.(A / p)^{-1-}\right)$ first mandibular molar tissues from $14 \mathrm{dpn}$ mice were compared by AB-NFR and PR staining. AB-NFR staining reveals a layer of nascent acellular cementum (AEFC) in (a and b) WT molar, while this layer was confirmed to be absent in the age-matched (c and d) $A / p I^{-1-}$ molar. PR staining under polarized light indicated that (e and f) collagen fibers were becoming organized in the WT molar PDL at this age; however, (g and $\mathbf{h}) \mathrm{Alp}^{-1-}$ molar PDL was severely disorganized with signs of periodontal detachment $(*)$ from the molar surface. Dotted boxes in panels $(\mathbf{a}),(\mathbf{c}),(\mathbf{e})$ and $(\mathbf{g})$ represent regions shown at higher magnification in panels (b), (d), (f) and (h), respectively. Original magnification was $\times 100$ for (a), (c), (e) and (g), and $\times 400$ for (b), (d), (f) and (h). AB-NFR, Alcian blue and nuclear fast red; AEFC, acellular extrinsic fiber cementum; dpn, days postnatal; PDL, periodontal ligament; PR, Picrosirius red; WT, wild-type.

\section{Comparative histology of cementum between species}

The mouse is the most commonly used model for studying tooth development; however, larger animal models or human specimens are sometimes used for developmental or repair studies, and can present quite different tissue dimensions and structure, as well as unique technical challenges in sample processing and staining. Pig and human molars were used for comparison of histological staining of the very different cementum structures in these species compared to mice.

Porcine molars feature a thick, cellular variety of cementum even on the cervical portion of the root, as previously described. ${ }^{30}$ The thickness varied from 80 to $150 \mu \mathrm{m}$ on the cervical half of the root, and numerous cementocytes were embedded in this thick tissue layer. $\mathrm{H} \& \mathrm{E}$ staining revealed gradations of pink coloration in dentin, cementum and PDL in the cervical pig molar (Figure 5a and 5b). Compared to the mouse molar cementum (Figure $2 \mathrm{a}-2 \mathrm{~d}$ ), pig cementum displayed little basophilia, appearing more similar to murine cellular cementum. Cementocytes as well as cells within the PDL displayed the expected basophilic nuclei. The CDJ was visible as a light pink zone (arrowheads in Figure 5a). With AB-NFR staining, the PDL and most of the dentin stained pink (Figure $5 \mathrm{e}$ and $5 \mathrm{f}$ ). The cementum also stained mostly pink; however, the initial cementum layer at the CDJ stained an intense blue in some regions (similar to AEFC in the mouse molar), and embedded cementocytes were also colored blue in their lacunae (Figure 5d). Underlying dentin was pale blue-purple and dentinal tubules were sometimes blue-tinged.

The human molar in Figure 6 featured a thick acellular cementum (approximately $50 \mu \mathrm{m}$ ) layer on the cervical root, and cellular or mixed stratified cementum (more than $400 \mu \mathrm{m}$ in some areas) on the apical and furcation regions of the root. $\mathrm{H} \& \mathrm{E}$ provided good contrast for identifying the initial cementum layer in the mixed stratified type, and highlighted the cementum annuli indicating annual apposition of this tissue over time (Figure 5c). H\&E marked the initial acellular cementum layer; however, the bulk of the cementum, dentin and PDL stained similar shades of blue in the human molar (Figure 5d). Like H\&E stain, AB-NFR was able to mark the initial cementum layer in mixed stratified cementum, and was present in dentin tubules as noted in mouse and pig (Figure $5 \mathrm{~g}$ ). Notably, by both $\mathrm{H} \& \mathrm{E}$ and $\mathrm{AB}-\mathrm{NFR}$ staining, few lacunae in human cellular cementum were occupied by cementocytes, though more cementocytes were present in 'newer' cementum regions closer to PDL (Figure 5g), a trend previously noted. ${ }^{3}$ AB-NFR marked the initial cementum layer in human acellular cementum with a brilliant blue band, and highlighted the cellular PDL region with pink counterstain, while dentin and PDL generally stained pale blue (Figure $5 \mathrm{~h}$ ).

In mouse, pig and human teeth in Figures 2 and 5, AB-NFR stained the initial layer of cementum on the cervical portions of teeth. While speculative, one might hypothesize that the intense blue coloration of the innermost cementum layer under $\mathrm{AB}-\mathrm{NFR}$ represents a secretion of ECM proteins accompanying initiation of cementogenesis. If this is so, then AB-NFR may be more sensitive than H\&E for detecting the initial ECM portion of cementum. This CDJ region has been indicated to be a unique transitional structure critical for cementogenesis and periodontal function, based on structural analyses and developmental studies in rodents and humans. ${ }^{5,31-36}$ The nature of the cementum ECM is explored in the next section and Figure 6.

A deep survey of the literature can reveal little-known or rarely reported stains that may prove very useful to exploration 
Pig
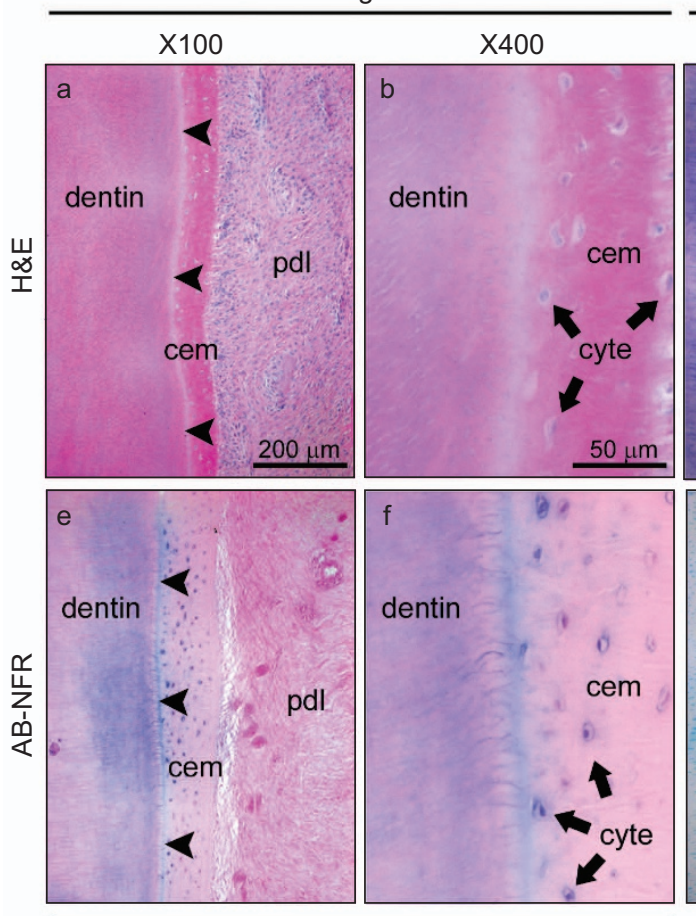

Cervical root cellular cementum
$\mathrm{X} 400$

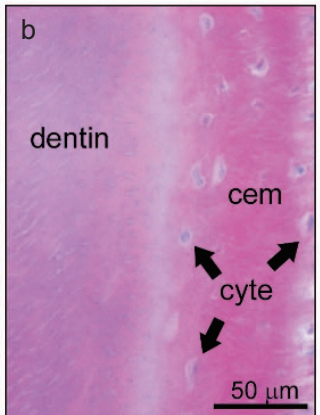

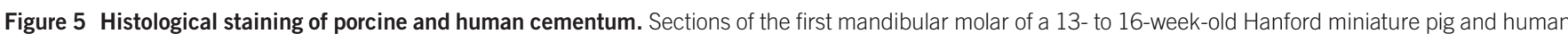

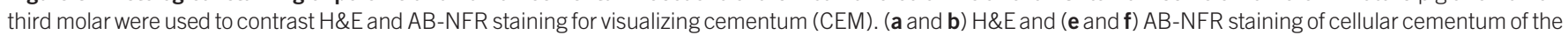

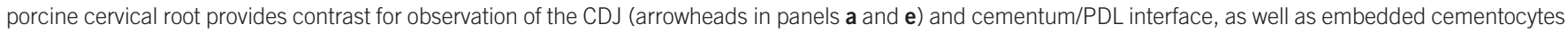

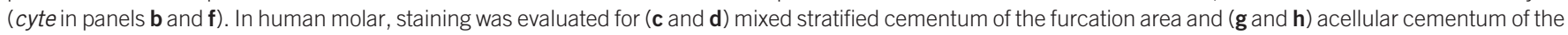

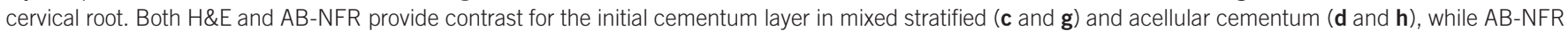

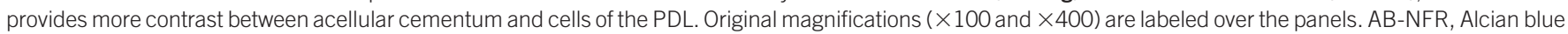
and nuclear fast red; CDJ, cementum-dentin junction; H\&E, hematoxylin and eosin; PDL, periodontal ligament.

Table 1 Promising staining techniques for visualizing cementum under light microscopy. The listed techniques that were chosen from among many in the literature that were indicated to provide useful differential staining of tooth root cementum, or that were suspected to provide such staining based on author's descriptions or images provided. Notes on the procedures and results are included, as well as references for full details

\begin{tabular}{|c|c|c|c|}
\hline Staining method & Species used & Notes & References \\
\hline $\begin{array}{l}\text { Gallego's iron fuschin stain, Lillie's } \\
\text { modification }\end{array}$ & Human teeth & $\begin{array}{l}\text { Calcified and undecalcified tissues can be used. } \\
\text { Cementum was stained in contrast to dentin and PDL. } \\
\text { Bone reversal lines were also stained positively }\end{array}$ & 37 \\
\hline Sudan black B & Rats, human fetal tissues & $\begin{array}{l}\text { Pyridine treatment is necessary prior to tissue processing. } \\
\text { Cementum was not explicitly studied; however, clear } \\
\text { patterns of staining of reversal lines in bone and mineralization } \\
\text { front in dentin appear to be present in cementum as well }\end{array}$ & 40 \\
\hline Procion dyes & $\begin{array}{l}\text { Mice, rats, rabbits, cats, } \\
\text { dogs and monkeys }\end{array}$ & $\begin{array}{l}\text { Twelve structurally related dyes were evaluated for their ability } \\
\text { to serve as vital dyes for mineralizing structures. Some were } \\
\text { capable of marking mineralization fronts in decalcified tissues } \\
\text { by light microscopy }\end{array}$ & 41 \\
\hline $\begin{array}{l}\text { Metal staining; Rio Hortega, Polak } \\
\text { mitochondria, and Achucarro- } \\
\text { Hortega tannic-silver methods }\end{array}$ & $\begin{array}{l}\text { Human teeth with } \\
\text { hypercementosis }\end{array}$ & $\begin{array}{l}\text { Several methods were compared for imaging hypercementosis, } \\
\text { with an emphasis on cementum shape, Sharpey's fiber insertion, } \\
\text { and cementocyte imaging }\end{array}$ & 38 \\
\hline $\begin{array}{l}\text { Fluorescent labeling with vital dyes } \\
\text { (tetracycline, calcein, alizarin) }\end{array}$ & Monkeys & $\begin{array}{l}\text { Cementum regeneration was tracked using vital dye dynamic } \\
\text { histomorphometry }\end{array}$ & 42 \\
\hline $\begin{array}{l}\text { Cresyl violet and periodic acid Schiff } \\
\text { staining methods }\end{array}$ & Human teeth & $\begin{array}{l}\text { Several stains were evaluated for their ability to mark cementum } \\
\text { incremental lines }\end{array}$ & 39 \\
\hline
\end{tabular}

of the cementum or other mineralized tissues. While we have not had the occasion to exhaustively try all these stains, and their detailed explanations are beyond the scope of this review, selected references ${ }^{37-42}$ are provided in Table 1, along with summaries of the staining techniques, in the case they may be of utility to readers. 

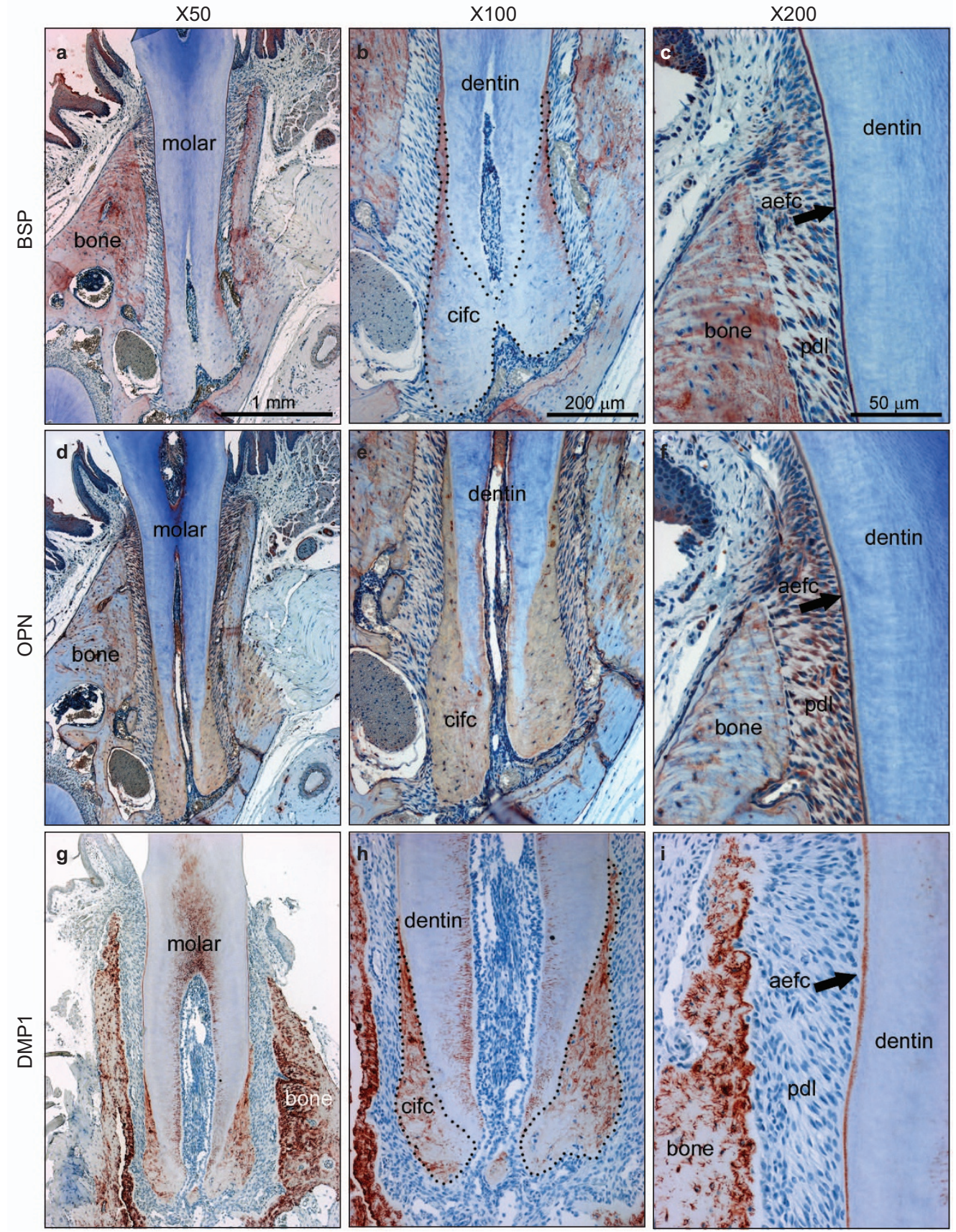

Figure 6 Immunohistochemistry for cementum. Mouse first mandibular molar sections at 44-45 dpn were immunostained for (a-c) BSP, (d-f) OPN and (G-I) DMP1. Both BSP and OPN localize to acellular and cellular cementum (AEFC and CIFC, respectively), as well as bone, though OPN is more widely distributed, such as in PDL. DMP1 localizes to the cementum-dentin junction region, as well as cellular cementum of the apex. A dotted line is used to highlight the circumference of the CIFC in panels $(\mathbf{b})$ and $(\mathbf{h})$. Original magnifications $(\times 50, \times 100$ and $\times 200)$ are labeled over the panels. AEFC, acellular extrinsic fiber cementum; BSP, bone sialoprotein; CIFC, cellular intrinsic fiber cementum; DMP1, dentin matrix protein 1; dpn, days postnatal; OPN, osteopontin; PDL, periodontal ligament.

\section{IHC for cementum}

While histological stains can highlight tissues or provide contrast for imaging, IHC has the potential to provide tissue specificity when antibodies are carefully chosen and employed. Both acellular and cellular cementum share many properties with bone, and no truly specific markers have been identified for these respective mineralized tissues. ${ }^{1,3,6}$ However, a small number of ECM proteins have proven useful both for imaging cementum and understanding its developmental biology. BSP, OPN and DMP1 are members of the small integrin binding ligand glycoprotein family, also including several other ECM proteins primarily associated with mineralized tissues. ${ }^{17}$ IHC for BSP revealed very strong localization in the thin AEFC layer, strong and even staining in bone, and diffuse localization to the thick CIFC layer, with little staining in the soft tissues (Figure 5a-5c). OPN, by contrast, was more widespread and also present in the PDL, but highlighted the AEFC as strongly as BSP (Figure $5 \mathrm{~d}-5 \mathrm{f}$ ). OPN was present in CIFC, providing good contrast from underlying dentin, and bone in particular displayed patterns indicating the presence of OPN 
at reversal or cement lines, an idea consistent with a role for OPN in cell-matrix interactions during remodeling. ${ }^{36,43-45}$ This concentration of BSP and OPN in AEFC supports the notion that these ECM proteins must be of critical importance in cementogenesis; ${ }^{35-36,46}$ however, their roles currently remain undefined. Still, these proteins have often been used to investigate the developmental biology of cementum and are useful as markers in that context. $3,6,9,12,35-36,47-49$

DMP1 was found at highest concentrations around osteocytes in bone, and could also be visualized in dentinal tubules (Figure 5g-5i). DMP1 was found in cellular cementum matrix, and especially focused around embedded cementocytes, in parallel fashion to osteocytes in bone (Figure $5 \mathrm{~h}$ ). While DMP1 has been reported to localize to acellular cementum in some species, ${ }^{50-51}$ our IHC labeled DMP1 at the CDJ and not throughout the entire cementum layer as BSP and OPN localize (Figure 5i, compared to Figure $5 \mathrm{c}$ and $5 \mathrm{f}$ ). The function of DMP1 in cementum is not understood at present, although localization of the protein, as well as transgenic mouse studies, ${ }^{50}$ suggests a potential role at the cementum/dentin interface, or cementum initiation. DMP1 immunostaining in mouse acellular cementum is often weak compared to BSP and OPN; therefore, it is not recommended as a primary marker for that tissue.

\section{CONCLUSIONS}

While cementum shares many properties in common with bone and dentin, existing data support it as a distinct and unique mineralized tissue. Further, there is more work to be done in order to understand the developmental biology of cementum, and how this knowledge can be brought to bear on approaches for cementum regeneration. Approaches for studying cementum development therefore become essential. Comparison of multiple histological stains revealed that while commonly applied $\mathrm{H} \& \mathrm{E}$ and TB stains offer adequate results, these are not necessarily optimal for cementum visualization. The infrequently used Alcian blue with nuclear fast red counterstain was found to provide excellent contrast for both acellular and cellular cementum in mouse, as well as in pig and human molars. PR staining viewed under polarized light provided insight on PDL collagen fiber insertion into the cementum surface, as well as PDL fiber bundle organization. While no truly unique ECM markers have been identified to differentiate cementum from the other hard tissues, IHC for BSP and OPN that localize to both acellular and cellular cementum layers is a reliable approach for visualizing cementum and providing insight into its developmental biology.

\section{ACKNOWLEDGEMENTS}

This research was supported (in part) by the Intramural Research Program of the National Institute of Arthritis and Musculoskeletal and Skin Diseases of the National Institutes of Health. Thanks to Dr Martha Somerman (NIAMS, NIH, Bethesda, MD, USA) and Dr Tracy Popowics (University of Washington School of Dentistry, Seattle, WA, USA) for critically reading and providing editorial suggestions on the manuscript. Thanks to Jirawan Wade (University of Washington School of Dentistry) for histological sectioning of mouse tissues. Thanks to Dr Tracy Popowics for preparing and providing porcine tissue samples, and Dr Kuang-Dah Yeh (University of Washington School of Dentistry) for porcine tissue processing and sectioning. Thanks to Dr José Luis Millán (Sanford Burnham Institute, La Jolla, CA, USA) for providing Alplmice. Thanks to Dr Francisco H Nociti and Cristiane R Salmon (Campinas State University, Piracicaba, SP, Brazil) for mouse tissues for DMP1 IHC.

1 Foster B, Popowics $\mathrm{T}$, Fong $\mathrm{H}$ et al. Advances in defining regulators of cementum development and periodontal regeneration. Curr Top Dev Biol 2007; 78: 47-126.
2 Diekwisch T. The developmental biology of cementum. Int J Dev Biol 2001; 45(5/6): 695-706.

3 Bosshardt D. Are cementoblasts a subpopulation of osteoblasts or a unique phenotype? J Dent Res 2005; 84(5): 390-406.

4 Bosshardt D, Selvig K. Dental cementum: the dynamic tissue covering of the root. Periodontol 2000 1997; 13: 41-75.

5 Bosshardt D, Schroeder H. Cementogenesis reviewed: a comparison between human premolars and rodent molars. Anat Rec 1996; 245(2): 267-292.

6 Foster BL, Somerman MJ. Cementum. In: McCauley LK, Somerman MJ eds. Mineralized tissues in oral and craniofacial science: biological principles and clinical correlates. Ames: Wiley-Blackwell, 2012: 169-192.

7 Everts V, Niehof A, Tigchelaar-Gutter W et al. Transmission electron microscopy of bone. Methods Mol Biol 2012; 816: 351-363.

$8 \mathrm{An} \mathrm{YH}$, Martin KL. Handbook of histology methods for bone and cartilage. Totowa: Humana Press, Inc, 2003.

9 Foster BL, Nagatomo KJ, Nociti FH et al. Central role of pyrophosphate in acellular cementum formation. PLoS ONE 2012; 7(6): e38393.

10 Narisawa S, Fröhlander N, Millán J. Inactivation of two mouse alkaline phosphatase genes and establishment of a model of infantile hypophosphatasia. Dev Dyn 1997; 208(3): 432-446.

11 Yeh KD, Popowics T. Molecular and structural assessment of alveolar bone during tooth eruption and function in the miniature pig, sus scrofa. Anat Histol Embryol 2011; 40(4): 283-291.

12 Foster BL, Nagatomo KJ, Bamashmous SO et al. The progressive ankylosis protein regulates cementum apposition and extracellular matrix composition. Cells Tissues Organs 2011; 194(5): 382-405.

13 Fong H, Chu EY, Tompkins KA et al. Aberrant cementum phenotype associated with the hypophosphatemic hyp mouse. J Periodontol 2009; 80(8): 1348-1354.

14 Cao Z, Zhang H, Zhou X et al. Genetic evidence for the vital function of Osterix in cementogenesis. J Bone Mineral Res 2012; 27(5):1080-1092.

15 Yamamoto T, Domon T, Takahashi S et al. The structure and function of the cementodentinal junction in human teeth. J Periodontal Res 1999; 34(5): 261-268.

16 Leong NL, Hurng JM, Djomehri SI et al. Age-related adaptation of bone-PDL-tooth complex: Rattus-Norvegicus as a model system. PLoS ONE 2012; 7(4): e35980.

17 Fisher L, Fedarko N. Six genes expressed in bones and teeth encode the current members of the SIBLING family of proteins. Connect Tissue Res 2003; 44(Suppl 1): 33-40.

18 Qin C, Baba O, Butler W. Post-translational modifications of sibling proteins and their roles in osteogenesis and dentinogenesis. Crit Rev Oral Biol Med 2004; 15(3): 126136.

19 Ho S, Kurylo M, Fong T et al. The biomechanical characteristics of the boneperiodontal ligament-cementum complex. Biomaterials 2010; 31(25): 6635-6646.

20 Junqueira L, Bignolas G, Brentani R. Picrosirius staining plus polarization microscopy, a specific method for collagen detection in tissue sections. Histochem J 1979; 11(4): 447-455.

21 Rodrigues TL, Nagatomo KJ, Foster BL et al. Modulation of phosphate/pyrophosphate metabolism to regenerate the periodontium. A novel in vivo approach. J Periodontol 2011; 82(12): 1757-1766.

22 Trombetta JM, Bradshaw AD. SPARC/osteonectin functions to maintain homeostasis of the collagenous extracellular matrix in the periodontal ligament. J Histochem Cytochem 2010; 58(10): 871-879.

23 Montes G, Junqueira L. The use of the Picrosirius-polarization method for the study of the biopathology of collagen. Mem Inst Oswaldo Cruz 1991; 86(Suppl 3): 1-11.

24 Beertsen W, McCulloch C, Sodek J. The periodontal ligament: a unique, multifunctional connective tissue. Periodontol 2000 1997; 13: 20-40.

25 Beertsen W, VandenBos T, Everts V. Root development in mice lacking functional tissue non-specific alkaline phosphatase gene: inhibition of acellular cementum formation. J Dent Res 1999; 78(6): 1221-1229.

26 McKee MD, Nakano Y, Masica DL et al. Enzyme replacement therapy prevents dental defects in a model of hypophosphatasia. J Dent Res 2011; 90(4): 470-476.

27 Millán J, Narisawa S, Lemire I et al. Enzyme replacement therapy for murine hypophosphatasia. J Bone Miner Res 2008; 23(6): 777-787.

28 Whyte M. Hypophosphatasia and the role of alkaline phosphatase in skeletal mineralization. Endocr Rev 1994; 15(4): 439-461.

29 van den Bos T. Handoko G, Niehof A et al. Cementum and dentin in hypophosphatasia. J Dent Res 2005; 84(11): 1021-1025.

30 Furseth R. A microradiographic, light microscopic and electron microscopic study of the cementum from deciduous teeth of pigs. Acta Odontol Scand 1970; 28(6): 811831.

31 Ho S, Yu B, Yun W et al. Structure, chemical composition and mechanical properties of human and rat cementum and its interface with root dentin. Acta Biomater 2009; 5(2): 707-718.

32 Ho SP, Balooch M, Goodis HE et al. Ultrastructure and nanomechanical properties of cementum dentin junction. J Biomed Mater Res A 2004; 68(2): 343-351.

33 Ho SP, Balooch M, Marshall SJ et al. Local properties of a functionally graded interphase between cementum and dentin. J Biomed Mater Res A 2004; 70(3): 480-489.

34 Bosshardt D, Schroeder $\mathrm{H}$. Initiation of acellular extrinsic fiber cementum on human teeth. A light- and electron-microscopic study. Cell Tissue Res 1991; 263(2): $311-$ 324.

35 Bosshardt D, Zalzal S, McKee M et al. Developmental appearance and distribution of bone sialoprotein and osteopontin in human and rat cementum. Anat Rec 1998; 250(1): 13-33. 
36 McKee M, Zalzal S, Nanci A. Extracellular matrix in tooth cementum and mantle dentin: localization of osteopontin and other noncollagenous proteins, plasma proteins, and glycoconjugates by electron microscopy. Anat Rec 1996; 245(2): 293-312.

37 Levey RJ. A modified Gallego's iron fuschin stain as a differential stain for cementum. J Dent Res 1956; 35(3): 491-493.

38 Comellilia RC, Lauand F, Marcantonio E et al. A contribution to the histological study of hypercementosis using metal staining. J Dent Res 1978; 57(1): 146-152.

39 Kvaal SI, Solheim T, Bjerketvedt D. Evaluation of preparation, staining and microscopic techniques for counting incremental lines in cementum of human teeth. Biotech Histochem 1996; 71(4): 165-172.

40 Irving JT, Wuthier RE. Further observations on the Sudan black stain for calcification. Arch Oral Biol 1961; 5: 323-324.

41 Prescott GH, Mitchell DF, Fahmy $\mathrm{H}$. Procion dyes as matrix markers in growing bone and teeth. Am J Phys Anthropol 1968; 29(2): 219-224.

42 Ogura N, Mera T, Sato F et al. Longitudinal observation of cementum regeneration through multiple fluorescent labeling. J Periodontol 1991; 62(4): 284-291.

43 Sodek J, Ganss B, McKee MD. Osteopontin. Crit Rev Oral Biol Med 2000; 11(3): 279-303.

44 D'Errico J, Sauk J, Prince C et al. Osteopontin adhesion receptors on gingival fibroblasts. J Periodontal Res 1995; 30(1): 34-41.

45 Somerman M, Shroff B, Foster R et al. Mineral-associated adhesion proteins are linked to root formation. Proc Finn Dent Soc 1992; 88(Suppl 1): 451-461.
46 MacNeil R, Berry J, D'Errico J et al. Role of two mineral-associated adhesion molecules, osteopontin and bone sialoprotein, during cementogenesis. Connect Tissue Res 1995; 33(1/2/3): 1-7.

47 Chu $\mathrm{E}$, Fong $\mathrm{H}$, Blethen $\mathrm{F}$ et al. Ablation of systemic phosphate-regulating gene fibroblast growth factor 23 (Fgf23) compromises the dentoalveolar complex. Anat Rec (Hoboken) 2010; 293(7): 1214-1226.

48 Fong $\mathrm{H}$, Chu EY, Tompkins KA et al. Aberrant cementum phenotype associated with the hypophosphatemic hyp mouse. J Periodontol 2009; 80(8): 1348-1354.

49 Viswanathan $\mathrm{H}$, Berry J, Foster B et al. Amelogenin: a potential regulator of cementumassociated genes. J Periodontol 2003; 74(10): 1423-1431.

$50 \mathrm{Ye} \mathrm{L}$, Zhang S, Ke $\mathrm{H}$ et al. Periodontal breakdown in the Dmp1 null mouse model of hypophosphatemic rickets. J Dent Res 2008; 87(7): 624-629.

51 Toyosawa S, Okabayashi K, Komori T et al. mRNA expression and protein localization of dentin matrix protein 1 during dental root formation. Bone 2004; 34(1): 124-133.

This work is licensed under a Creative Commons

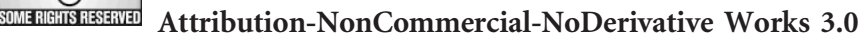
Unported License. To view a copy of this license, visit http:// creativecommons.org/licenses/by-nc-nd/3.0 